\title{
Inion Not a Reliable Landmark of the Torcula in Posterior Fossa Craniotomies
}

\author{
Elisha Harry Otieno, Philip Mwachaka, Paul Odula, Isaac Cheruiyot, Jeremiah Munguti
}

School of Medicine, University of Nairobi, Nairobi, Kenya

Correspondence to: Elisha Harry Otieno; email: collinharry09@gmail.com.

\begin{abstract}
Background: Sub-occipital craniotomies are used in surgical approaches into the posterior cranial fossa. The inion is used as an extracranial landmark of the torcula in burr hole placement. However, inadvertent damage to the torcula and the related sinuses due to variant intracranial location of the torcula has been reported. This study aimed at determining the position of the torcula in relation to the inion. Method: 40 adult open skulls were obtained and the positional relationship between the inion and the torcula assessed. Results: The torcula was at the same level with the inion in half of the cases $(50 \%)$, below it in 12 cases (30\%), and above in 8 cases $(20 \%)$. The position above the inion ranged from 0.38 to $2.40 \mathrm{~cm}$ and below it from 0.75 to $2.45 \mathrm{~cm}$. Conclusion: The torcula lies either at the level of the inion in only half
\end{abstract}

\section{Introduction}

Craniotomy is a basic implement of a neurosurgeon's surgical practice and is indicated for a number of neuropathologies. Posterior cranial fossa (PFC) craniotomies in particular are often employed for surgical treatment of a wide array of tumors including fourth ventricular tumors, medulloblastomas, astrocytomas and pineal region tumors $(1,2)$. Indeed, PFC tumors are the most prevalent of all neuro-oncologies (3). Treatment of other conditions such as epidural hematoma, Ponto bulbar injuries, brain cysts and Chiari I malformations also necessitate the procedure.

PFC craniotomies date back to the end of the 19th century when relatively wide exposures of the fossa were performed. These included mastoid-to-mastoid lateral incisions and cross-bow incisions on the occipital bone, which were frequently associated with high mortality rates of up to $71 \%$ (4). Consequently, a single vertical midline incision, involving bone resection from the lower border of the external occipital protuberance to the posterior arch of the foramen magnum, was adopted (5). of the cases. The surgeon should augment this physical landmark with radiographs to avoid iatrogenic injuries.

Keywords: Inion, Posterior fossa, Craniotomies

Ann Afr Surg. 2020;17(3):103-105

DOI: http://dx.doi.org/10.4314/aas.v17i3.3

Conflicts of Interest: None

Funding: None

(C) 2020 Author. This work is licensed under the Creative Commons Attribution 4.0 International License

Submitted: 11 November 2019

Revised: 12 February 2020

Accepted: 6 March 2020

Online first: 29 May 2020

This incision has been widely considered a safer procedure and is still practiced to date. However, with the advent of modern equipment, the current standard technique involves drilling and joining two burr holes on either side of the midline just inferior to the transverse sinuses (6).

Despite the fairly complex evolution of this surgical procedure, inadvertent injury to underlying intracranial venous sinuses, frequently involving the torcula, is still reported (7). The torcula, also referred to as the confluence of sinuses, is the junction of superior sagittal, straight and transverse Dural venous sinuses in the occipital region of the skull. It is documented in anatomical literature to lie on the internal occipital protuberance (IOP), which is thought to correspond to the external occipital protuberance (EOP) extracranially. While imaging has been shown to improve neurosurgical evaluation of cerebral drainage pathways and transverse sinus dominance, it is still not well documented as to how imaging can be employed to locate the torcula using external landmarks (8). Thus, neurosurgeons use the 
inion, the prominent projection of the external occipital protuberance, as a reference to estimate the position of the torcula. Drilling a burr hole below the inion would presumably avoid direct damage to the torcula. However, iatrogenic accidents have been reported, suggesting a possible intracranial variation in the torcula's position versus the inion. This variation is supported by a previous cadaveric study that showed that the IOP corresponded to the inion in just 2 out of 15 specimens (9). Continual use of the inion by neurosurgeons despite this finding piqued our interest to determine whether there are populationspecific variations of the anatomical position of the torcula.

\section{Materials and methods \\ Study design and setting}

This was a descriptive cross-sectional study using 40 adult crania of both sexes. The crania were from formalin-fixed cadavers used in routine dissection. The data were collected in March 2018. Skulls that exhibited obvious deformities were excluded from the study. Permanent markers, dissecting forceps, a manual vernier caliper (accurate to $0.01 \mathrm{~cm}$ ), and a 13MP digital camera were used to expose the specimen and collect data. Permission for this study was provided by the Human Anatomy Department, University of Nairobi.

\section{Technique}

A pair of forceps was positioned with one arm reaching the center of the torcula on the inside and the other outside the skull, and this extra-cranial position marked. The inion was then located on the occiput and similarly marked. The vertical distance between these two points was measured using a vernier caliper and recorded (Fig. $1 \mathrm{~A} \& \mathrm{~B})$.

\section{Results}

The torcula was at the same level with the inion in half of the cases (50\%), below the inion in 12 cases (30\%), and

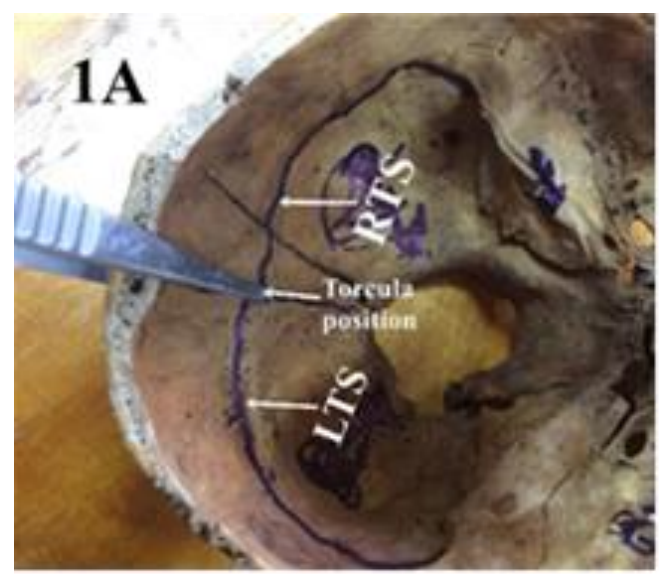

Figure $1 \mathrm{~A}$. Measuring the torcula above it in 8 cases (20\%). The average position above the inion was $1.39 \mathrm{~cm}$ and below it $1.60 \mathrm{~cm}$. The highest inion was $1.39 \mathrm{~cm}$ and below it $1.60 \mathrm{~cm}$. The highest position of the torcula in relation to the inion was $2.40 \mathrm{~cm}$ while the lowest position was $2.45 \mathrm{~cm}$.

\section{Discussion}

Extracranial landmarks are important guides for craniotomy during posterior fossa surgery. This is because the advent of modern preoperative imaging techniques has not been shown to significantly improve patient outcomes. Thus, this anatomical knowledge remains essential. This particularly applies to junior medical and surgical trainees who are yet to amass surgical experience for these procedures, and those practicing in low-income countries where the number of trained radiologists is inadequate. This knowledge will thus aid safe burr hole drilling on the occiput without adverse complications.

There is general paucity of data on the torcula-inion relationship. A study in a Turkish population reported the torcula to range from 1.1 to $2.3 \mathrm{~cm}$ below the inion (10). Different results were presented in a similar study (9), noting that the torcula lay above the inion in almost $80 \%$ of the cases studied. The inion is not synonymous with the EOP, but actually its projected apex. The findings of this study further complicate the debate. Possibility of modality and methodological differences could account for this disparity in the findings. This includes defining and determining the "center" of either the anatomical points used and the morphological differences in sizes of the torcula. Moreover, adaptational changes of the musculoskeletal tissue around the occiput could create these variant positions of the inion.

The variant location of the torcula could be attributed to an array of developmental and pathological factors. It is

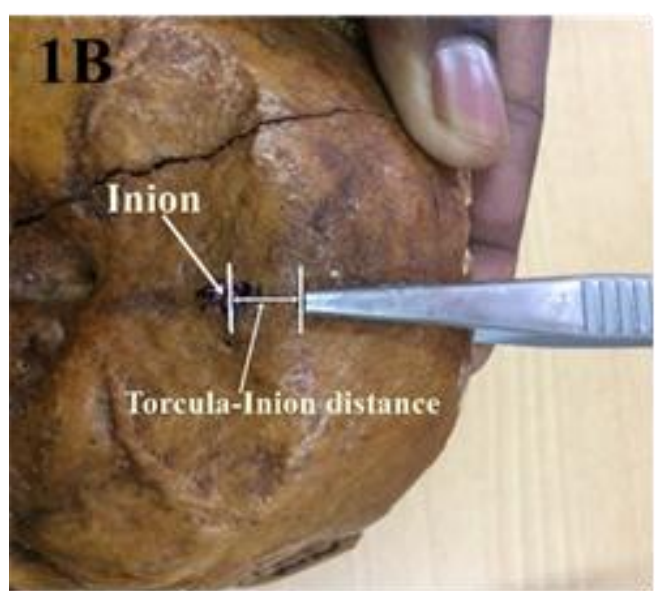

Figure 1B. Measuring the torcula-inion distance 
well known that the EOP bears no functional relation to the IOP. Whereas the IOP and the transverse sulci housing the transverse sinus owe their prominence to constant hemodynamic erosion from venous blood, the EOP and their inion particularly gain prominence due to pull from cranial and cervical musculature, which could have significant inter-individual variations (9). Because these muscular attachments are established in utero, inevitable age-related differences in cranial position further dispute the reliability of the landmark for infants or younger children. Pathological states such as cystic fluid accumulation in the posterior fossa or fetal malformations, such as arachnoid cyst, Dandy-Walker syndrome, and a mega cisterna magna (11), could also displace the dural sinuses to a higher cranial level, and the surgeon needs to be aware of this.

Evidently, there is need for further studies on the topic, especially imaging studies that would eliminate bias from the use of manual instruments as well as the selection for reference point.

\section{Conclusion}

Our findings show that the torcula lies at the level of the inion in half of the cases examined. This is however inconclusive. Absolute dependence on extracranial landmarks when performing craniotomy should be avoided and, whenever possible, preoperative evaluation of the surgical patient using radiography should also be adopted to lower risks of iatrogenic injury.

\section{References}

1. Lee HC, Lee JY, Ryu SK, et al. A method of posterior fossa dural incision to minimize hemorrhage from the occipital sinus: The "mosquito" method. Childs Nerv Syst ChNS Off J Int Soc Pediatr Neurosurg. 2016;32(12):2429-31.

2. Grover K, Sood S. Midline suboccipital burr hole for posterior fossa craniotomy. Childs Nerv Syst ChNS Off J Int Soc Pediatr Neurosurg. 2010;26(7):953-5.

3. Kushel YV, Sorokin VS, Chel'diev BZ, et al. Surgery of posterior cranial fossa tumors in children in the prone position. The surgical technique features. Zh Vopr Neirokhir Im N Burdenko. 2018;82(3):36-41.

4. Viale G. The surgical approach to the posterior cranial fossa according to Galen. Neurosurgery. 2007;61(5 Suppl 2):399_ 403.

5. Bucy PC. Exposure of the posterior or cerebellar fossa. J Neurosurg. 1966;4(4):820-32.

6. Giammattei L, Borsotti F, Parker F, et al. Chiari I malformation: Surgical technique, indications and limits. Acta Neurochir (Wien). 2018;160(1):213-7.

7. Oh GS, Arnone GD, Abou-Al-Shaar H, et al. Surgical repair of iatrogenic transverse-sigmoid sinus laceration with a dural flap during skull base tumor surgery: A technical case report. World Neurosurg. 2017;106:1050. e7-1050.e10.

8. Bayaroğulları H, Burakgazi G, Duman T, et al. Evaluation of dural venous sinuses and confluence of sinuses via MRI venography: anatomy, anatomic variations, and the classification of variations. Childs Nerv Syst. 2018; 34(6):1183-8.

9. Tubbs RS, Salter G, Oakes WJ, et al. Superficial surgical landmarks for the transverse sinus and torcularherophili. J Neurosurg. 2000;93(2):279-81.

10. Ziyal IM, Ozgen T, Tubbs RS, et al. Landmarks for the transverse sinus and torcularherophili. J Neurosurg. 2001;94(4):686-7.

11. Shekdar K. Posterior fossa malformations. Semin Ultrasound CT MR. 2011;32(3):228-41. 